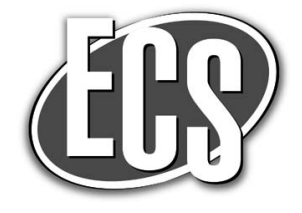

JSS Focus Issue on Nanocarbons in Sensing Applications

\title{
Toward Practical Non-Contact Optical Strain Sensing Using Single-Walled Carbon Nanotubes
}

\author{
Peng Sun, ${ }^{a}$ Sergei M. Bachilo, ${ }^{\mathrm{b}}$ Satish Nagarajaiah, ${ }^{\mathrm{a}, \mathrm{c}}$ and R. Bruce Weisman ${ }^{\mathrm{b}, \mathrm{c}, *, \mathrm{z}}$ \\ ${ }^{a}$ Department of Civil and Environmental Engineering, Rice University, Houston, Texas 77005, USA \\ ${ }^{b}$ Department of Chemistry, Rice University, Houston, Texas 77005, USA \\ ${ }^{c}$ Department of Materials Science and NanoEngineering, Rice University, Houston, Texas 77005, USA
}

\begin{abstract}
Progress is reported in an emerging non-contact strain sensing technology based on optical properties of single-walled carbon nanotubes (SWCNTs). In this strain-sensing smart skin (" $\mathrm{S}^{4}$ ") method, nanotubes are dilutely embedded in a thin polymer film applied to a substrate of interest. Subsequent strain in the substrate is transferred to the nanotubes, causing systematic spectral shifts in their characteristic short-wave infrared fluorescence peaks. A small diode laser excites a spot on the coated surface, and the resulting emission is captured and spectrally analyzed to deduce local strain. To advance performance of the method, we prepare $\mathrm{S}^{4}$ films with structurally selected SWCNTs. These give less congested emission spectra that can be analyzed precisely. However, quenching interactions with the polymer host reduce SWCNT emission intensity by an order of magnitude. The instrumentation that captures SWCNT fluorescence has been made lighter and smaller for hand-held use or mounting onto a positioning mechanism that makes efficient automated strain scans of laboratory test specimens. Statistical analysis of large $\mathrm{S}^{4}$ data sets exposes uncertainties in measurements at single positions plus spatial variations in deduced baseline strain levels. Future refinements to $S^{4}$ film formulation and processing should provide improved strain sensing performance suitable for industrial application.

(C) The Author(s) 2016. Published by ECS. This is an open access article distributed under the terms of the Creative Commons Attribution Non-Commercial No Derivatives 4.0 License (CC BY-NC-ND, http://creativecommons.org/licenses/by-nc-nd/4.0/), which permits non-commercial reuse, distribution, and reproduction in any medium, provided the original work is not changed in any way and is properly cited. For permission for commercial reuse, please email: oa@electrochem.org. [DOI: 10.1149/2.0031608jss] All rights reserved.
\end{abstract}

Manuscript submitted January 22, 2016; revised manuscript received February 18, 2016. Published February 26, 2016. This paper is part of the JSS Focus Issue on Nanocarbons in Sensing Applications.

In structural health monitoring, routine inspection and maintenance are vital to ensure the safe operation and maximum service life of critical structures, including bridges, high-rise building supports, oil platforms, pipelines, pressure vessels, and airframes. Strain measured on the surface of any structure provides direct first-hand information about its health condition. Established strain sensor technologies, such as resistance strain gages and fiber Bragg grating (FBG) sensors, and some newer ones, including piezoelectric sensors, ${ }^{1}$ MEMS doubleended tuning fork (DETF) strain gauges, ${ }^{2}$ and nanotube films, ${ }^{3}$ are all point-wise, unidirectional sensing methods that require physical connections to the sensor elements in order to obtain strain readings. They are therefore classified as contact strain sensing methods. By contrast, the focus of this report is a new technology for noncontact strain sensing.

Some existing full-field non-contact optical methods have also been developed, such as interferometric techniques, ${ }^{4}$ noninterferometric techniques, ${ }^{5-7}$ and Raman spectroscopy. ${ }^{8}$ The interferometric techniques, including holography, interferometry, speckle interferometry, etc., usually require a model of actual structure, tedious calculations to separate the values of principal stresses, and expensive equipment. Non-interferometric techniques like the grid method, ${ }^{5}$ and digital image correlation (DIC) ${ }^{6,7}$ suffer from some other limitations, such as the requirements for a random gray intensity distribution or speckle pattern distribution, heavy dependence on the quality of the imaging system, relatively low strain measurement accuracy in small deformation measurements, and the inability to measure strains induced when the object is not directly observed by the imaging system. Over the past two decades, Raman spectroscopy has been explored by many researchers in strain sensing applications. ${ }^{8-12}$ However, Ramanbased strain sensing approaches are badly hampered by the low intensity of Raman scattering signals. They therefore need impractically long acquisition times to achieve acceptable signal-to-noise ratios in real field applications.

To enable effective non-contact strain sensing at arbitrary locations and along arbitrary axes on surfaces of interest, we are de-

\footnotetext{
*Electrochemical Society Fellow.
}

${ }^{\mathrm{z}}$ E-mail: weisman@ rice.edu veloping a technology called strain-sensing smart skin, or $\mathrm{S}^{4} \cdot{ }^{13-15}$ This method is based on the unique spectroscopy of semiconducting single-walled carbon nanotubes (SWCNTs). SWCNTs are a family of artificial nanomaterials with remarkable physical properties and many potential uses. ${ }^{16}$ Each nanotube is a cylindrical tubular structure composed of covalently bonded carbon atoms. SWCNT diameters are typically near $1 \mathrm{~nm}$ and lengths after processing are generally between 200 and $2000 \mathrm{~nm}$. SWCNTs comprise a variety of distinct transverse structures with long-range crystalline order. Each of these is labeled by a pair of integers, $(n, m)$, and has well-defined diameter, roll-up (chiral) angle, and $\pi$-electron energy levels determined by quantum confinement. Most SWCNT structures are semiconducting and show $(n, m)$-dependent bandgaps separating the filled valence states from vacant conduction states. These semiconducting SWCNTs can be excited with visible light to induce spectrally sharp bandgap fluorescence emission in the short-wave infrared (SWIR) spectral region between $\sim 900$ and $1600 \mathrm{~nm} .{ }^{17}$ SWCNT absorption and emission wavelengths are precisely known as a function of $(n, m)$ structure from the spectroscopic assignment studies of Bachilo, Weisman, and coworkers. ${ }^{18,19}$ Because nanotube growth reactors produce mixtures of many species, as-grown samples show a variety of spectral transitions spanning a range of wavelengths. However, recent progress in postgrowth sorting of mixed samples enables the extraction of SWCNT fractions that are highly enriched in any of several $(n, m)$ species and have dramatically simpler emission spectra.

When a semiconducting SWCNT is deformed by compression or stretching along its axis, positions of the carbon atoms move in systematic patterns and make the electronic bandgap larger or smaller. This shifts the wavelength of the nanotube's SWIR emission peak. It has been shown theoretically and experimentally that the magnitudes and signs of these spectral shifts are proportional to strain along the nanotube axis and are well described by the following expression. ${ }^{20,21}$

$$
|\Delta \mathrm{E}|=3 \mathrm{t}_{0}(1+\nu) \cos (3 \theta) \cdot \varepsilon
$$

Here $\Delta \mathrm{E}$ is the strain-induced energy shift of the fluorescence photon, $\mathrm{t}_{0}$ is an electron hopping energy parameter; $v$ is the SWCNT Poisson ratio; $\theta$ is the SWCNT roll-up angle (which may range from $0^{\circ}$ to $30^{\circ}$ ), and $\varepsilon$ is the axial strain. 

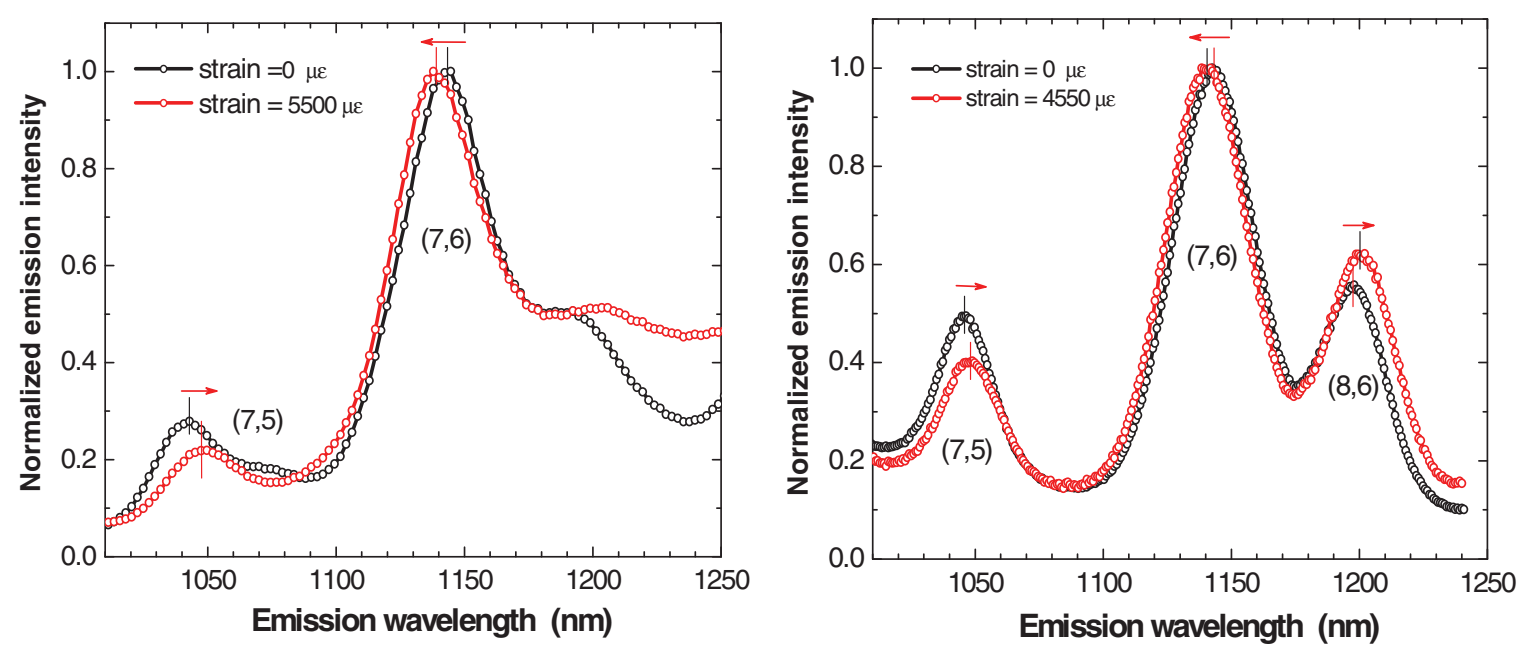

Figure 1. Strain-induced changes in emission spectra for Generation-I $S^{4}$ film (left panel) and Generation-II $S^{4}$ film (right panel). The excitation wavelength was $660 \mathrm{~nm}$.

Strain-induced spectral shifts are greatest for small roll-up angles (near-"zigzag") and smallest for large angles (near-"armchair"). Another important feature is that the peaks of one class of semiconducting SWCNTs (the " $\bmod 1$ " nanotubes, for which $\bmod (n-m, 3)=$ 1) shift down in wavelength when they are axially stretched while the peaks of the other class (" $\bmod 2 ")$ shift oppositely under the same deformation. This behavior suggests monitoring strain from the difference between spectral positions of a $\bmod 1$ peak and a $\bmod 2$ peak in a structurally mixed nanotube sample. Such spectral difference measurements should effectively cancel out small systematic shifts in SWCNT peak emission wavelengths caused by changes in the dielectric environment if there are drifts in the properties of the host polymer.

Another important feature of SWCNT spectroscopy is that the optical transitions for visible excitation and SWIR emission are both strongly polarized along the nanotube axis. So when polarized excitation light irradiates a film containing randomly oriented nanotubes, it will selectively reveal strain along the polarization axis because it preferentially excites the subset of nanotubes aligned in that direction. Therefore simple rotation of the polarization plane allows one to map strain versus direction on the surface being monitored.

Over the past few years, we have made progress toward developing a practical technology in which these SWCNT optical properties are applied to let them serve as nanoscale strain sensors when embedded in thin polymeric films applied to surfaces of interest. ${ }^{13-15}$ In this $\mathrm{S}^{4}$ approach, strain in the coated substrate is transmitted through the film, causing axial strains in embedded nanotubes. We then quantitatively monitor the nanotube strain at any position of interest by irradiating that spot with a visible laser of suitable wavelength and capturing the resulting SWIR fluorescence for spectral interpretation. The axis of strain can be determined by varying the laser's polarization plane. The $\mathrm{S}^{4}$ method promises noncontact strain sensing with improved simplicity and versatility for structural health monitoring.

Our first-generation smart skin composite was prepared by dispersing raw SWCNTs into commercial urethane varnish. Although these Generation-I $\mathrm{S}^{4}$ films showed promising results for strain sensing, ${ }^{13}$ their fluorescence spectra are congested from the presence of emission peaks from numerous $(n, m)$ species (see Figure 1). This hampers the extraction of precise peak positions and shifts. In order to avoid this problem, we developed Generation-II S ${ }^{4}$ films, in which the SWCNTs were pre-processed by selective extraction to provide structural sorting and purification. ${ }^{15}$ As shown in Figure 1, such Generation-II $\mathrm{S}^{4}$ films display more structured fluorescence spectra, allowing precise measurements of several $(n, m)$ peak positions and the use of combined peak shifts for improved strain determinations. We report here recent results in the development of the $\mathrm{S}^{4}$ method for practical non-contact strain sensing.

\section{Materials and Instrumentation}

The process for preparing Generation-II S $\mathrm{S}^{4}$ films is illustrated in Figure 2. It uses a commercial urethane varnish (Minwax Helmsman

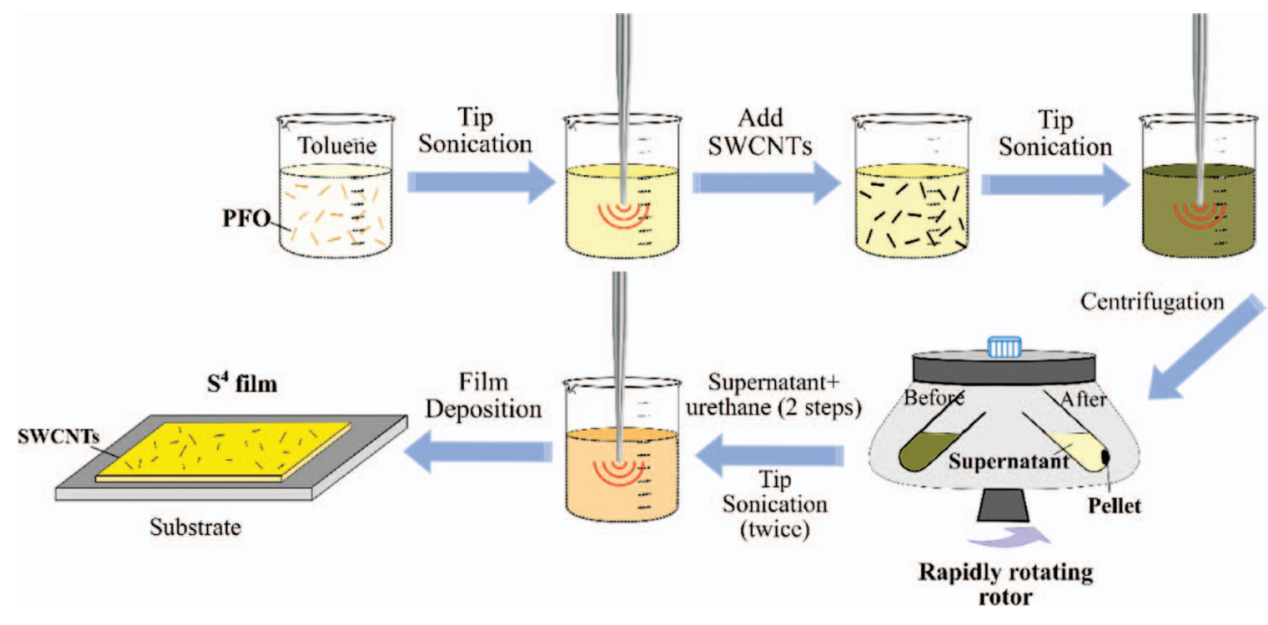

Figure 2. Protocol for preparing Generation-II $\mathrm{S}^{4}$ films. 

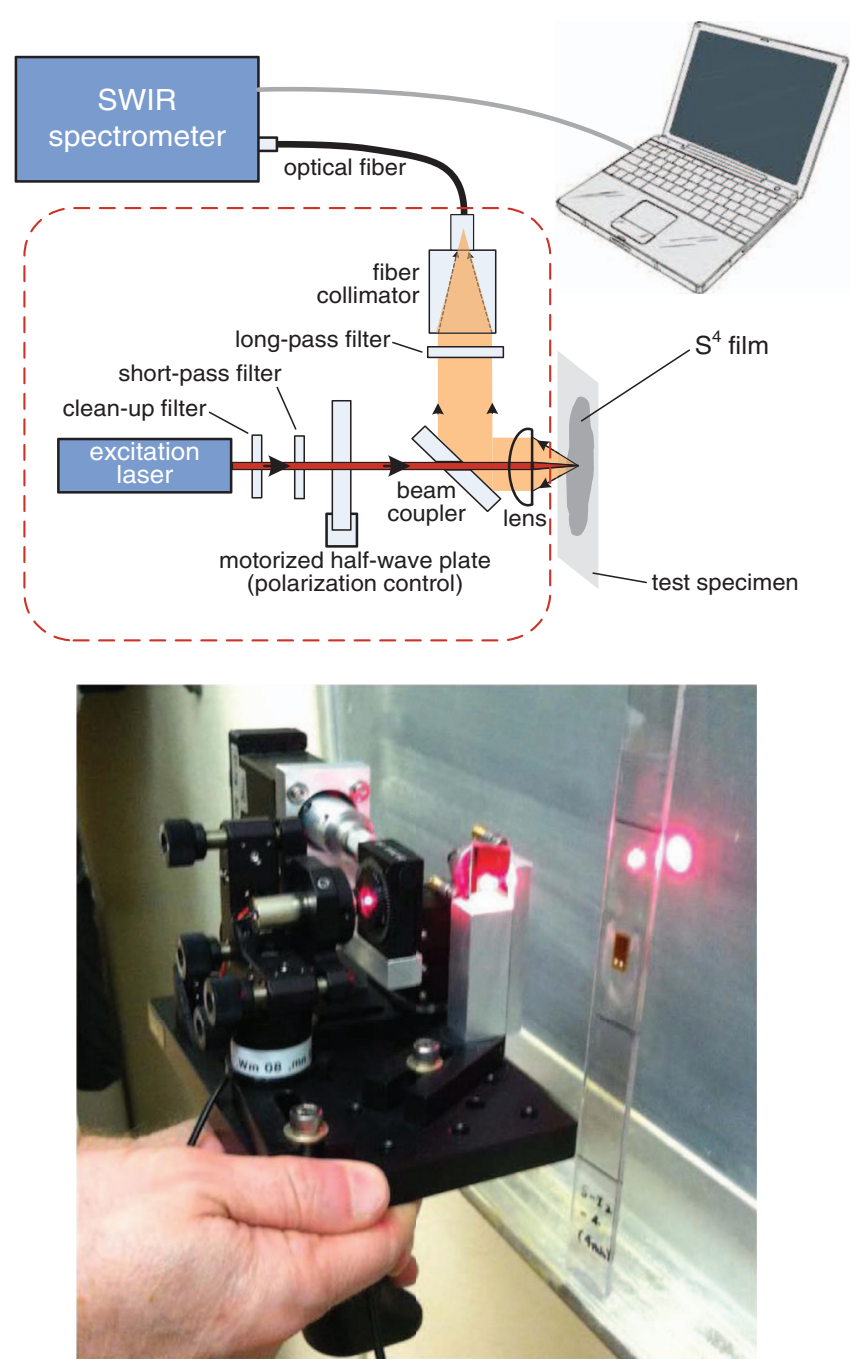

Figure 3. Top: Schematic diagram of prototype $\mathrm{S}^{4}$ strain probe. All components within the dashed red line are mounted on a $4^{\prime \prime} \times 6^{\prime \prime}$ plate. Bottom: Photo of the $4^{\prime \prime} \times 6^{\prime \prime}$ plate with probe optical components.

Spar Urethane, 350 VOC compliant) as the polymeric host and SWCNTs grown in the Rice University HiPco reactor as strain sensors. The raw, bundled nanotubes are first extracted into a toluene solution of the organic dye PFO (poly(9,9-di-n-octylfluorenyl-2,7-diyl). This process individualizes and selectively wraps and suspends a small subset of the $(n, m)$ species. ${ }^{22}$ Solid PFO is added to toluene at a mass concentration of $1 \mathrm{mg} / \mathrm{mL}$ and dissolved with the aid of $60 \mathrm{~min}$ of tip ultrasonication at a power of $1 \mathrm{~W} / \mathrm{mL}$ and a duty cycle of $20 \mathrm{~s}$ on, $40 \mathrm{~s}$ off (conditions used for all ultrasonication treatments in this work). Raw SWCNTs are then added to the PFO/toluene solution at a concentration of $0.1 \mathrm{mg} / \mathrm{mL}$. The mixture is ultrasonicated for $45 \mathrm{~min}$ to obtain a suspension with homogeneous appearance and then centrifuged at $25^{\circ} \mathrm{C}$ for $30 \mathrm{~min}$ at $13,000 \mathrm{RPM}$ to pellet out impurities and remaining bundles. The supernatant containing disaggregated, extracted SWCNTs coated with PFO is carefully pipetted out and added to the urethane at a volume ratio of 1:1. After tip ultrasonication of the composite for $6 \mathrm{~min}$, more urethane is added to increase its volume by $50 \%$ and it is ultrasonicated for another 6 min. As illustrated in Figure $1, \mathrm{~S}^{4}$ films made with PFO-extracted SWCNTs show emission spectra with superior clarity as compared to our Generation-I films. These processed samples are highly enriched in individualized SWCNTs, and any remaining bundled nanotubes are expected not to affect our results because they should undergo internal energy transfer after excitation that will either quench their emission

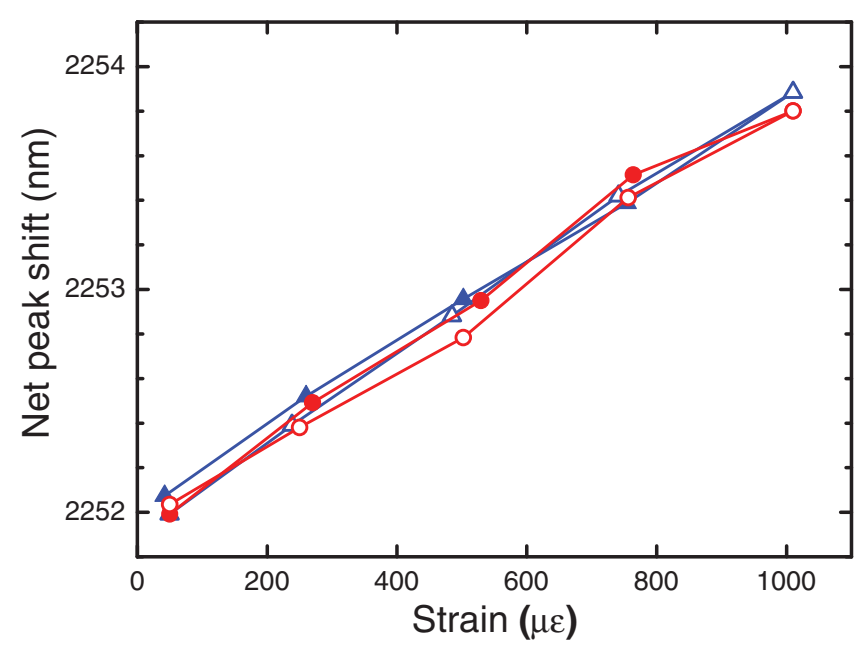

Figure 4. Measured spectroscopic peak shift parameter $((7,5)+(8,6))$ from a Generation-II $\mathrm{S}^{4}$ film vs. independently measured strain in the PMMA substrate. Data are shown for two loading/unloading cycles.

or red-shift it beyond the relevant spectral range. The SWCNTs in our processed samples have mean lengths near $400 \mathrm{~nm}$. Because this is approximately 100 times shorter than the measured SWCNT persistence length, ${ }^{23}$ we expect very little bending of nanotubes in the $\mathrm{S}^{4}$ film.

Practical application of the $\mathrm{S}^{4}$ method for non-contact strain sensing requires an optical readout apparatus that is compact and portable enough for field use. To this end, we have constructed a prototype reader head incorporating a small diode laser emitting $70 \mathrm{~mW}$ at 660 $\mathrm{nm}$ and optics for focusing the excitation beam onto the specimen surface, collecting resulting SWCNT emission, and performing appropriate spectral filtering, polarization control, and beam splitting. Figure 3 shows the schematic diagram and photograph of this prototype apparatus. SWCNT fluorescence captured by the reader head is transmitted through an optical fiber to a compact, modular multichannel InGaAs spectrometer, which records its spectrum and transmits data to a notebook computer for analysis. Details of the reader apparatus have been reported previously. ${ }^{15}$ We note that interference from ambient light can be avoided in field applications by placing an opaque baffle tube between the reader lens and the specimen surface. Also, ambient temperature thermal emission from surfaces is negligible at the SWIR wavelengths relevant for our method.

\section{Method Performance}

The Generation-II composite material described above was applied as a liquid to various test substrates and allowed to cure to give $\mathrm{S}^{4}$ films approximately 10 to $20 \mu \mathrm{m}$ thick. Spectra from SWCNTs in the film were then measured using the prototype readout apparatus while the specimen was subjected to controlled loading in tension. Actual induced strains were measured with conventional resistive foil gages for comparison with the observed changes in spectral peak positions. Figure 4 shows an example of the correlation found between spectral response of the $\mathrm{S}^{4}$ film and the independently measured deformation of a PMMA test specimen that was strained between 0 and $1000 \mu \varepsilon$ during two cycles of loading and unloading. The response is highly linear in this range, although at higher strains we observe deviations attributed to slippage of nanotubes in the host film. The upper limit to strains measureable by the $\mathrm{S}^{4}$ method will be determined by the strength of interfacial adhesion between SWCNTs and the surrounding polymer.

As described above, the $\mathrm{S}^{4}$ method can also determine the axis of strain. We illustrate this in Figure 5, which shows data measured for the spectral response (difference between the $(7,6)$ and $(7,5)$ peak wavelengths) at one point on a test specimen that was strained to 


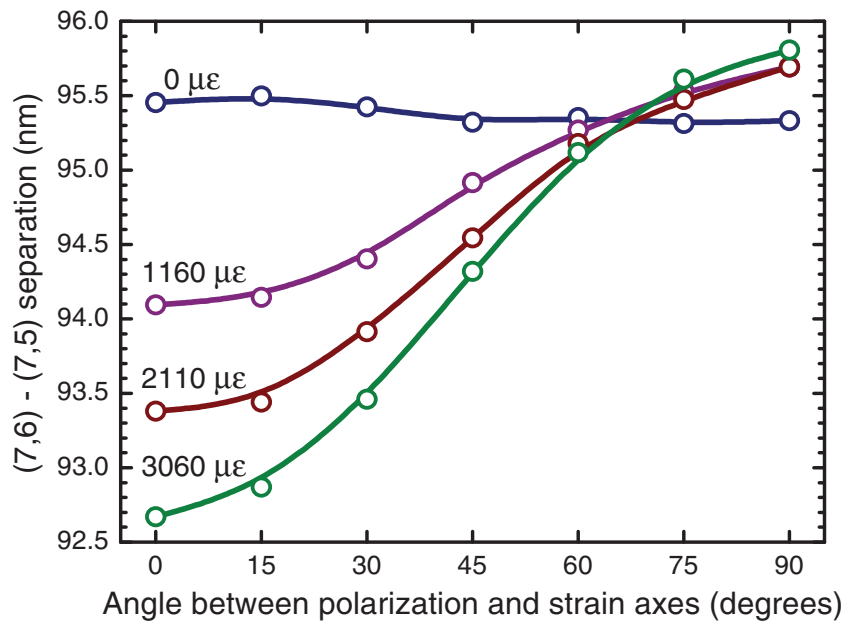

Figure 5. Points show the $(7,6)$ - $(7,5)$ peak separation from an $S^{4}$ film as a function of excitation beam polarization direction. Tension was applied to the test substrate along the zero-degree direction to give the three different labeled strains. Solid curves are best fits to $\cos ^{2}$ functions with adjusted amplitudes.

different extents along a single axis. For each strain level there is a smooth and systematic variation of spectral response as a function of the polarization direction of the excitation laser beam. The maximum responses are found at 0 degrees, which is the actual strain axis. It is clear then that the $\mathrm{S}^{4}$ method can determine directions as well as magnitudes of substrate strains.

Practical implementation requires the capture of high quality emission spectra to allow precise determination of small strain-induced changes in peak positions. In addition, these spectra must be acquired quickly to enable the preparation in reasonable amounts of time of strain maps derived from measurements at many locations on a surface. These constraints imply the need for spectra with high signalto-noise ratios, so optimization of fluorescence signal strengths is important. However, we have found (unexpectedly) that the SWCNT emission intensity is substantially reduced by quenching interactions between SWCNTs and the urethane host material. To investigate this quenching effect, we have measured emission spectra of a standard preparation of PFO-extracted SWCNTs after mixing with different amounts of the commercial spar urethane varnish. As shown in Figure 6, the emission intensity of SWCNT peaks decreases monotonically and dramatically after 1 volume of SWCNT suspension is added to $0.5,1,1.5$, or 2 volumes of urethane and mixed by 2 min of tip ultrasonication. Note that these spectra have been scaled to account for volumetric dilution, so the decreases indicate a strong reduction in the nanotubes' fluorescence quantum yield. To determine whether this effect might result from SWCNT damage in the ultrasonication process, we prepared equivalent mixtures with the varnish in which manual shaking was applied instead of tip ultrasonication. The results, plotted in Figure 7, show fluorescence quenching that is nearly as severe. Table I lists the normalized fluorescence intensities measured at

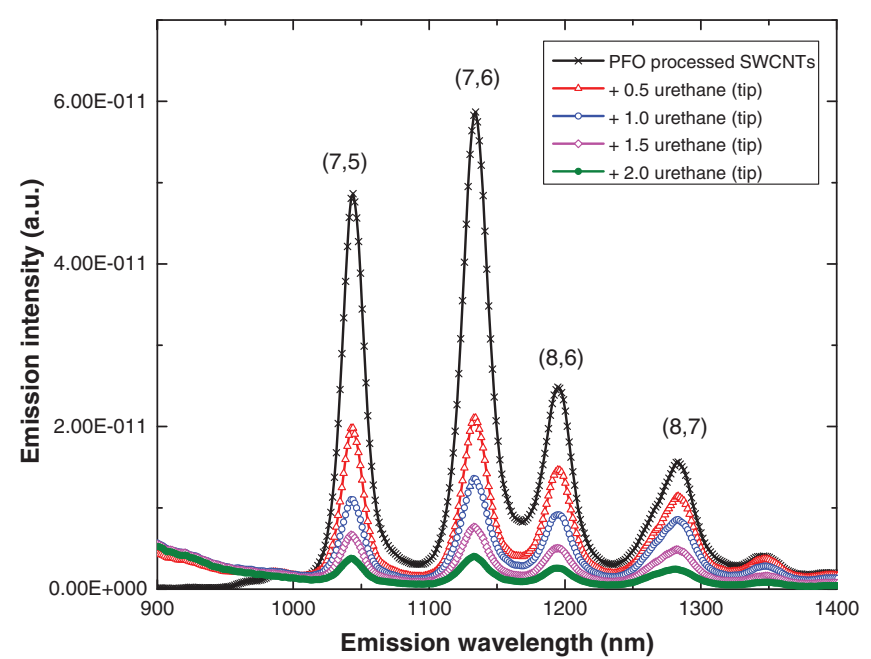

Figure 6. Emission spectra of PFO-SWCNTs mixed with spar urethane by tip sonication. The data have been scaled to compensate for the effects of dilution.

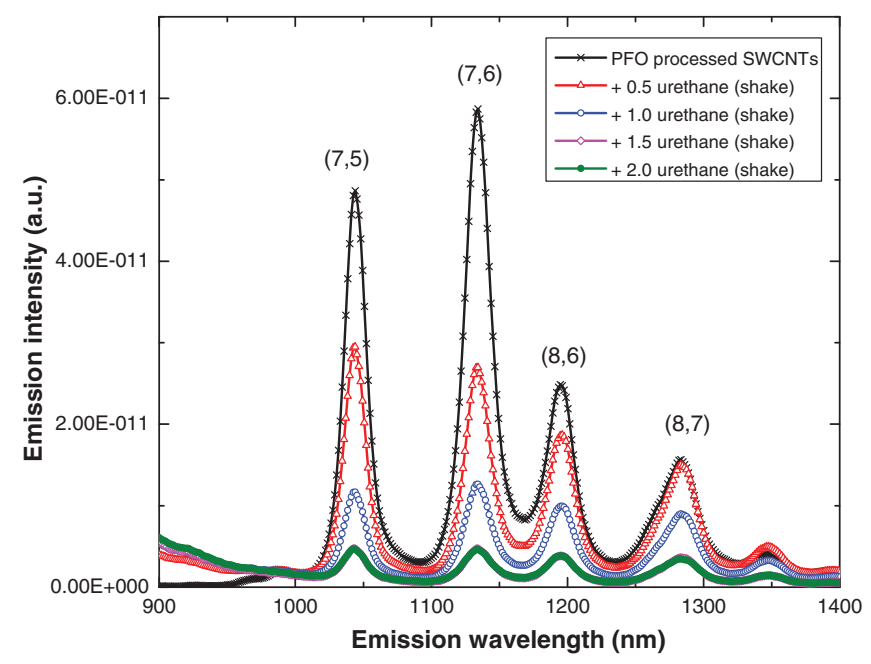

Figure 7. Emission spectra of PFO-SWCNTs mixed with spar urethane by shaking. The data have been scaled to compensate for the effects of dilution.

four different $(n, m)$ emission peaks after mixing with four different varnishes. It is found that all of the varnish compositions cause emission quenching by at least $83 \%$, with the smaller diameter nanotubes most strongly affected. This is consistent with a chemical interaction mechanism that is facilitated by the higher reactivity of small diameter SWCNTs caused by curvature bond strain. We conclude that different polymeric host materials or new film structures should be explored to optimize the emissive efficiency of $\mathrm{S}^{4}$ coatings.

Table I. Ratios of emission intensities from liquid suspensions of SWCNTs in PFO/toluene mixed with polymeric varnishes in 1:2 volume ratios, relative to emission intensities before mixing with varnish. Values are corrected for volumetric dilutions. "Tip" and "shake" denote mixing by tip ultrasonication and mechanical shaking, respectively.

Final / initial emission intensity

\begin{tabular}{|c|c|c|c|c|c|}
\hline \multirow[b]{2}{*}{ Commercial varnish } & \multirow[b]{2}{*}{ Main components } & \\
\hline & & $(7,5)$ & $(7,6)$ & $(8,6)$ & $(8,7)$ \\
\hline Minwax Spar urethane (shake) & aliphatic hydrocarbon solvent, urethane & $9.6 \%$ & $7.9 \%$ & $15.4 \%$ & $22.2 \%$ \\
\hline Minwax Fast-dying polyurethane (shake) & aliphatic hydrocarbon solvent, polyurethane & $4.9 \%$ & $4.7 \%$ & $8.6 \%$ & $9.5 \%$ \\
\hline Duralux Marine Spar Varnish (shake) & resin, mineral spirits (aliphatic and aromatic solvents) & $4.3 \%$ & $4.2 \%$ & $6.6 \%$ & $7.5 \%$ \\
\hline
\end{tabular}




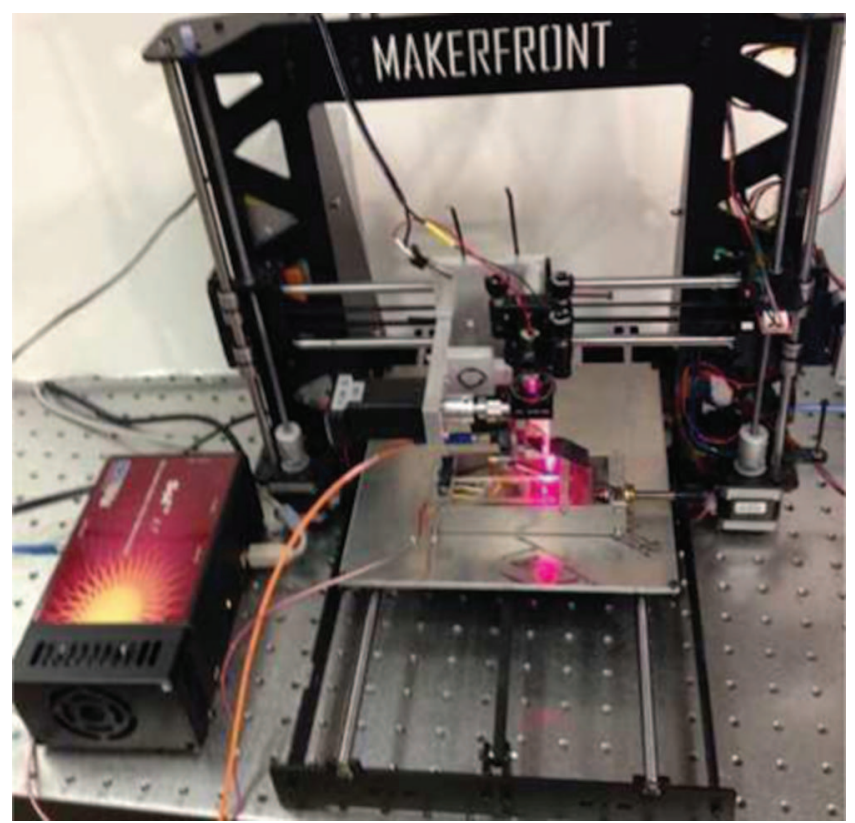

Figure 8. Photo of the $\mathrm{S}^{4}$ probe head mounted onto a computer-controlled 3D printer frame to allow automated scanning of specimens. The orange optical fiber transmits fluorescence collected from the $\mathrm{S}^{4}$ film to the SWIR spectrometer seen in the lower left of the photo.

\section{Automated Strain Scanning}

Automation of the $\mathrm{S}^{4}$ strain measurements is an important goal for two reasons. First, it can speed method development by allowing large data sets to be collected and statistically analyzed in reasonable amounts of time. This enables us to identify factors that limit the method's performance and quantitatively evaluate improvement efforts. Second, automation is clearly essential for practical implementation and surface strain mapping. $\mathrm{S}^{4}$ automation in the development lab involves both hardware and software. We need a hardware mechanism to accurately position and scan the optical read head under computer control across test specimens in order to assess film homogeneity and measure strains at specific locations. For this we have adapted a small commercial 3D printer by replacing its print head assembly with a version of our optical read head that is still lighter and more compact than the one in Figure 3. A photo of this scanning optical strain reader is shown in Figure 8. A notebook computer running custom LabVIEW software controls positioning of the read head and spectral data acquisition from the multichannel InGaAs spectrometer. Through stepper motor controllers, the program moves the read head to the desired $\mathrm{x}, \mathrm{y}$-coordinates (within a range of $200 \times 200 \mathrm{~mm}$ ) and then adjusts the $\mathrm{z}$-coordinate (height) for proper optical distance from the specimen surface. This process allows measurements on tilted or curved specimens. Positioning resolution is better than $0.1 \mathrm{~mm}$ in all axes. We note that the gage length for $\mathrm{S}^{4}$ measurements is smaller than $0.1 \mathrm{~mm}$. It is determined by the spot size of the focused excitation beam and is independent of film thickness. After the emission spectrum has been captured at a point, the program precisely locates the specific $(n, m)$ emission peaks selected for strain monitoring - typically $(7,5),(7,6)$ and $(8,6)$ - by fitting to separate local quadratic functions.

Measurement uncertainties in $\mathrm{S}^{4}$ strain map data may be caused by the limited precision of single-point strain readings and also by point-to-point differences in $\mathrm{S}^{4}$ film properties, such as inhomogeneous initial strains. The automated scanning system described above allows us to explore and distinguish these contributions. Figure 9 shows a histogram of the $(7,6)$ peak wavelengths found from 1650 repeated spectral measurements at the same position on an $\mathrm{S}^{4}$ film. The results fit very well to a Gaussian distribution with a full width at half-maximum of $0.35 \mathrm{~nm}$, corresponding to $\sim 100 \mu \varepsilon$ in deduced

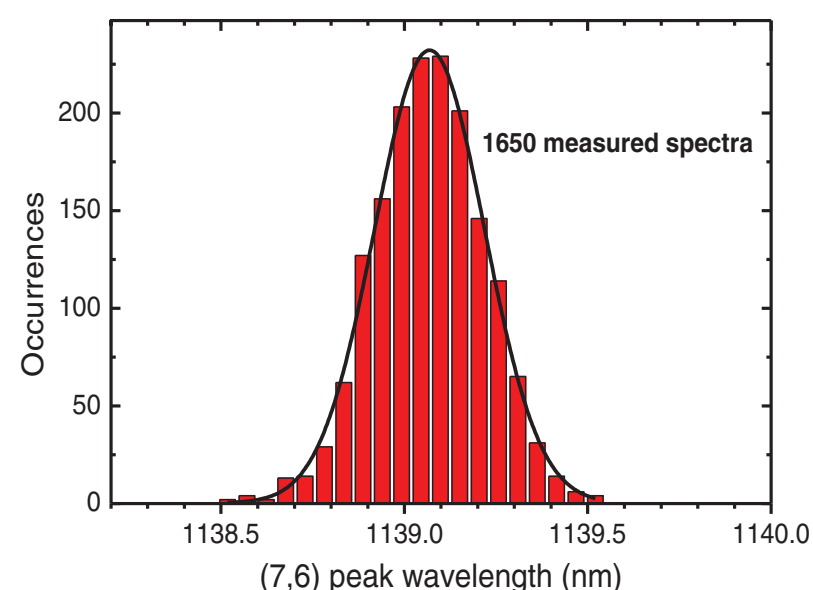

Figure 9. Histogram of $(7,6)$ peak positions deduced from 1650 repeated spectral measurements at one position on an $\mathrm{S}^{4}$ test film. The solid line is a Gaussian best fit to the data.

strain. We would then expect that $S^{4}$ measurements made at different points on a specimen surface would show variations in spectral peak positions given by this value plus a contribution from spatial inhomogeneity. The latter might result from local strain differences in uneven polymer films induced by contraction during curing. Using the automated scanning system and $2.5 \mathrm{~s}$ of data acquisition per position, we have performed spectral measurements at a grid of 169 positions on the surface of an $\mathrm{S}^{4}$-coated test plate before it was deliberately strained. Values found for the $(7,6)-(7,5)$ peak separation, which is proportional to strain, are plotted as a 2D color map in Figure 10. One can see that this baseline strain map shows minor systematic patterns that slightly exceed the single-point measurement uncertainty. The standard deviation for the entire set of 169 points is $0.23 \mathrm{~nm}$. Future experiments will explore ways to minimize the underlying spectral variations by optimizing coating formulations, surface pretreatments, application techniques, and film curing methods. We also hope to improve the precision of peak wavelength measurements by increasing the signal-to-noise ratio of captured spectra. This may be achieved by using future generation $\mathrm{S}^{4}$ films that avoid strong quenching of SWCNT fluorescence and provide more intense spectra, and/or by increased averaging of spectral data.

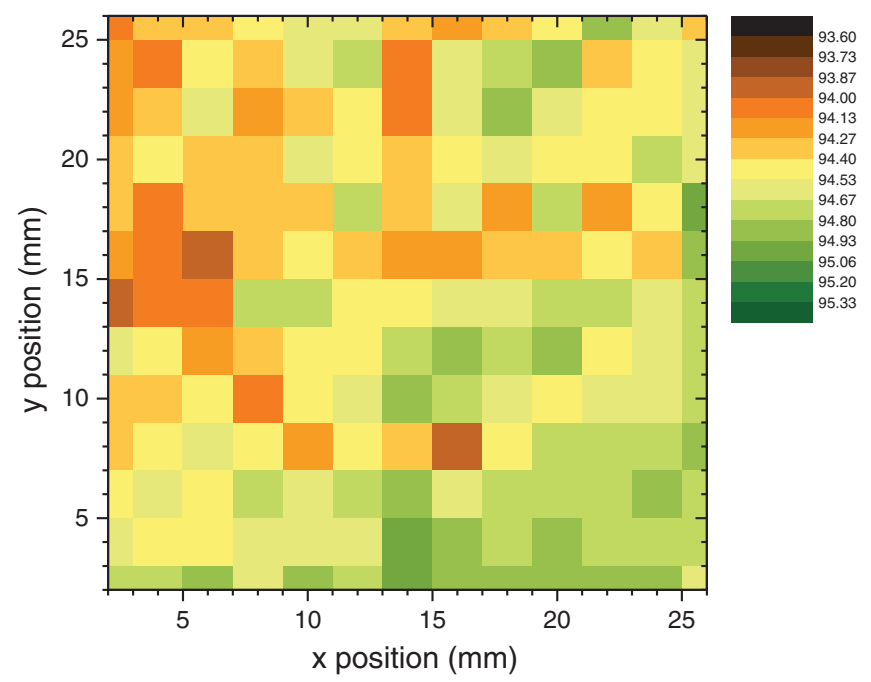

Figure 10. Plot of spectral strain parameters measured by scanning 169 points on the surface of a substrate coated with an $\mathrm{S}^{4}$ film. Note that this is a baseline map measured before the specimen was strained. Color-mapped values show separations (in $\mathrm{nm}$ ) between $(7,6)$ and $(7,5)$ spectral peaks. The standard deviation of the full set of points is $0.23 \mathrm{~nm}$. 


\section{Conclusions}

We have demonstrated that the unique intrinsic spectroscopic properties of single-walled carbon nanotubes allow them to serve as nanoscale strain sensors when embedded into thin polymeric coatings. This emerging non-contact measurement technology, termed $\mathrm{S}^{4}$, can find strain magnitude and direction at any point on a coated surface. Progress has been made in several areas needed for practical implementation of the $\mathrm{S}^{4}$ method. One is the development of coatings containing structurally selected nanotubes, which give less congested emission spectra that can be more precisely analyzed for strain signatures. In addition, the instrumentation needed to excite and capture nanotube fluorescence from $\mathrm{S}^{4}$ films has been made more efficient, compact, and lighter to enable strain readers to be scanned over stationary structural elements of interest. For laboratory studies on small test specimens, we have mounted such a reader head onto the positioning mechanism of a 3D printer and automated the data acquisition and analysis process. This permits the efficient collection of large data sets that quantitatively reveal measurement uncertainties and can guide further refinements. We have combined the improved $\mathrm{S}^{4}$ films, lightweight reader head, and automated positioning and data analysis to demonstrate the capability for basic 2D strain mapping of test surfaces. Future work should focus on reformulation of the $\mathrm{S}^{4}$ films to avoid strong SWCNT fluorescence quenching by components in the host polymer. This will enhance signal strengths to allow faster data scans and better spectral precision. Another goal is improved spatial uniformity of $\mathrm{S}^{4}$ film properties. Changes to the film formulation, application, and curing methods should be explored for this purpose. Assuming that it will be possible to address these issues, we think that the $\mathrm{S}^{4}$ method can become a practical and valuable new tool for structural health maintenance in many industrial applications.

\section{Acknowledgments}

This project has been supported by the Office of Naval Research (award ONR N00014-14-1-0013) and the Welch Foundation (award
C-0807). We thank C.-W. Lin for his generous help with the LabVIEW programming and the scanning system and J. Zhao for assistance with mechanical testing.

\section{References}

1. Y. Yong, IEEE/ASME Trans., 18, 1113 (2013).

2. R. G. Azevedo, D. G. Jones, A. V. Jog, B. Jamshidi, D. R. Myers, L. Chen, X. Fu, M. Mehregany, M. B. Wijesundara, and A. P. Pisano, IEEE Sens. J., 7, 568 (2007).

3. P. Dharap, Z. Li, S. Nagarajaiah, and E. V. Barrera, Nanotechnology, 15, 379 (2004)

4. A. Ajovalasit, G. Petrucci, and M. Scafidi, Strain, 46, 137 (2010).

5. H. Goldrein, S. Palmer, and J. Huntley, Opt. Lasers Eng., 23, 305 (1995)

6. B. Pan, Opt. Eng., 46, 033601 (2007).

7. S. Hamada, T. Fujisawa, M. Koyama, N. Koga, N. Nakada, T. Tsuchiyama, M. Ueda, and H. Noguchi, Mater. Charact., 98, 140 (2014).

8. Q. Zhao, M. D. Frogley, and H. D. Wagner, Compos. Sci. Technol., 62, 147 (2002).

9. Z. Li, P. Dharap, S. Nagarajaiah, E. V. Barrera, and J. D. Kim, Adv. Mater, 16, 640 (2004).

10. M. Mu, S. Osswald, Y. Gogotsi, and K. I. Winey, Nanotechnology, 20, 335703 (2009).

11. A. de la Vega, I. A. Kinloch, R. J. Young, W. Bauhofer, and K. Schulte, Compos. Sci. Technol., 71, 160 (2011)

12. A. P. A. Raju, A. Lewis, B. Derby, R. J. Young, I. A. Kinloch, R. Zan, and K. S. Novoselov, Adv. Funct. Mater, 24, 2865 (2014).

13. P. A. Withey, V. S. M. Vemuru, S. M. Bachilo, S. Nagarajaiah, and R. B. Weisman, Nano Lett., 12, 3497 (2012).

14. P. Sun, J.-H. Kim, S. M. Bachilo, R. B. Weisman, and S. Nagarajaiah, in SPIE Smart Structures and Materials + Nondestructive Evaluation and Health Monitoring, vol. 9061, p. 906120 (2014).

15. P. Sun, S. M. Bachilo, R. B. Weisman, and S. Nagarajaiah, J. Strain Anal. Eng. Des., 50, 505 (2015).

16. S. Reich, C. Thomsen, and J. Maultzsch, Carbon nanotubes: basic concepts and physical properties, John Wiley \& Sons, (2008).

17. M. J. O'Connell, S. M. Bachilo, C. B. Huffman, V. C. Moore, M. S. Strano, E. H. Haroz, K. L. Rialon, P. J. Boul, W. H. Noon, C. Kittrell, J. Ma, R. H. Hauge, R. B. Weisman, and R. E. Smalley, Science, 297, 593 (2002).

18. S. M. Bachilo, M. Strano, C. Kittrell, R. H. Hauge, R. E. Smalley, and R. B. Weisman, Science, 298, 2361 (2002).

19. R. B. Weisman and S. M. Bachilo, Nano Lett., 3, 1235 (2003).

20. T. K. Leeuw, D. A. Tsyboulski, P. N. Nikolaev, S. M. Bachilo, S. Arepalli, and R. B. Weisman, Nano Lett., 8, 826 (2008).

21. L. Yang and J. Han, Phys. Rev. Lett., 85, 154 (2000).

22. A. Nish, J.-Y. Hwang, J. Doig, and R. J. Nicholas, Nat. Nanotechnol., 2, 640 (2007).

23. N. Fakhri, D. A. Tsyboulski, L. Cognet, R. B. Weisman, and M. Pasquali, Proc. Natl. Acad. Sci. USA, 106, 14219 (2009). 